\title{
Hypothermic preservation of corneas in a hyperkalaemic solution (CPTES): I. Short-term storage in the absence of colloid osmotic agents
}

\author{
MICHAEL J TAYLOR AND CHARLES J HUNT \\ From the MRC Medical Cryobiology Group, University Department of Surgery, Cambridge CB2 $2 A H$
}

SUMmaRY Preservation solutions for short-term storage of isolated donor corneas for use in penetrating keratoplasty have all been based on tissue culture medium, on the assumption that media designed to maintain the viability of cells at physiological temperatures will also provide suitable conditions for preservation at reduced temperatures. But for hypothermic preservation of some other tissues and organs, when ionic pumps are inhibited, it is unnecessary to support metabolism, and beneficial control of ion and water distribution between intra- and extracellular compartments is achieved by storage in appropriately formulated 'intracellular-type' solutions. We have therefore designed a solution that will restrict ionic imbalances and minimise endothelial cell swelling in corneas during exposure at reduced temperatures. This potassium-rich solution contains the biological $\mathrm{pH}$ buffer TES as an impermeant anion and is designated CPTES (cornealpotassium-TES). The structural and functional integrity of rabbit corneas stored at $0^{\circ} \mathrm{C}$ in CPTES, without the addition of colloid osmotic agents, is compared with that of corneas stored in glutathione bicarbonate Ringers' solution (GBR), an 'extracellular-type' medium formulated for the maintenance of endothelial integrity during in-vitro perfusion at $34^{\circ} \mathrm{C}$. Corneas swelled significantly less during storage in CPTES than in GBR and could be stored for five days before reaching the same degree of hydration as corneas stored for only three days in GBR. Gross structural integrity and endothelial ultrastructure were maintained during storage for three and five days in CPTES. The rate of thinning of corneas stored in CPTES was significantly greater than in comparable groups of corneas stored in GBR. However, the efficient dehydration of corneas stored in CPTES was always preceded during perfusion by a brief period of additional swelling which was shown to be an osmotic response during the elution of the buffer compound TES that had permeated the stroma during storage. The omission of calcium or the addition of adenosine and glutathione to the CPTES preservation medium had no detectable effect on the integrity of the endothelium, but the omission of bicarbonate was beneficial, producing significantly higher rates of stromal thinning during normothermic perfusion. Additional benefits for extending storage by including colloid osmotic agents are described in a companion paper.

Preservation of some biological tissues at reduced temperatures succeeds because cooling prolongs invitro survival by slowing metabolism, reducing the demand for oxygen and other metabolites, and by

Correspondence to $\operatorname{Dr}$ M J Taylor, MRC Medical Cryobiology Group, University Department of Surgery, Douglas House, Trumpington Road. Cambridge CB2 $2 \mathrm{AH}$. conserving chemical energy.' Historically the short term preservation of corneas has been achieved by storing enucleated eyes in the humid environment of a so-called moist chamber at $4^{\circ} \mathrm{C}$. The accepted time limit imposed on this method of storage is $24-48 \mathrm{~h} .^{2}$ However, storage of corneas while still an integral part of the eyeball has been questioned, because 
post-mortem changes in the composition of the aqueous humour are known to make it unsuitable, even toxic, as a medium for bathing the endothelium during storage. ${ }^{3-5}$ Furthermore, swelling of the cornea during $4^{\circ} \mathrm{C}$ storage makes it difficult to achieve contiguity of the graft with the host bed. Such problems have led to the practice of excising the cornea and storing it in a suitable medium separated from the eyeball.

However, adoption of storage techniques in clinical practice has often proceeded in advance of the establishment of a firm specification for what constitutes a suitable medium for hypothermic storage of corneas. In the past decade preservation solutions have been based on TC199 tissue culture medium with the addition of either dextran (M-K medium), ${ }^{6}$ polyvinylpyrrolidone, ${ }^{7}$ or glycosaminoglycans $(\mathrm{K}-\mathrm{SOL})^{\times 9}$ as oncotic agents to limit the degree of tissue oedema during storage. With this approach effective preservation of corneas for penetrating keratoplasty has been extended to about one week. "' However, the particular balance of ions and molecules in tissue culture medium is designed to maintain the metabolic status of cells in vitro at physiological temperature and therefore will not be optimal for maintaining the ionic and hydraulic balance within corneal endothelium at reduced temperatures.

An approach that has proved beneficial in the hypothermic storage of organs and other tissues assumes that, at reduced storage temperatures, it is unnecessary to support metabolism, and that the normal distribution of water and ions between the intracellular and extracellular compartments can be maintained by physical rather than metabolic means.' Since during the period that metabolic pumps are inactivated the driving force for sodium loading and potassium depletion from the cells is the difference in ionic balance between the intraand extracellular fluid, and the driving force for water uptake (cell swelling) is the impermeant intracellular anion, these changes should be prevented or restricted by immersion of the tissue in an appropriate 'intracellular-type' solution.

With this objective in mind we have advocated the use of a hyperkalaemic balanced salt solution (CPTES) containing an impermeant anionic buffer, $\mathrm{N}$-tris (hydroxymethyl) methyl-2-aminoethane sulphonic acid (TES), as an appropriate bathing medium for the low temperature preservation of corneas. "The sulphonic acid buffer, TES, is one of a family of zwitterionic compounds which have found widespread use as highly effective biological $\mathrm{pH}$ buffers at both physiological and reduced temperatures. ${ }^{12-14}$ The potassium-rich CPTES solution containing 100 millimolar TES was designed primarily as a suitable medium for adding and removing cryoprotectants during procedures for the long term cryopreservation of corneas, and has been shown to be an effective 'vehicle' solution for adding and removing multimolar concentrations of dimethyl sulphoxide in isolated rabbit corneas at $0^{\circ} \mathrm{C} .1115$ The purpose of this investigation was to evaluate the merits of CPTES as a solution for intermediate-term storage at $0^{\circ} \mathrm{C}$ in the absence of cryoprotective agents.

In this paper the structural and functional integrity of corneal endothelium is examined after one or three days' storage at $0^{\circ} \mathrm{C}$ in CPTES and is compared with that of corneas stored under similar conditions in glutathione bicarbonate Ringer's solution (GBR), and 'extracellular-type' balanced salt solution formulated for the maintenance of endothelial integrity during in-vitro perfusion at $34^{\circ} \mathrm{C} .11$ in 17 Endothelial integrity was also examined after five days' storage in CPTES at $0^{\circ} \mathrm{C}$. Parameters studied in the evaluation of corneal integrity at the end of storage include the initial stromal thickness immediately following storage, corneal hydration changes during in-vitro perfusion on the specular microscope, and corneal endothelial cell ultrastructure.

Studies which examine extended storage in CPTES with the addition of colloid osmotic agents are described elsewhere. ${ }^{18} 19$

\section{Material and methods}

Corneas were excised from the enucleated eyes of New Zealand White rabbits $(2-3 \mathrm{~kg})$ as previously described. "For storage at $0^{\circ} \mathrm{C}$ polypropylene vials containing $5 \mathrm{ml}$ of the preservation medium were placed in an evacuated Dewar flask containing ice which was itself placed in a $4^{\circ} \mathrm{C}$ refrigerator for the storage period. Isolated corneas, supported on plastic rings, were immersed endothelial side uppermost in the precooled storage solution. At the end of the storage interval corneas were either mounted in the perfusion chamber of a specular microscope to assess endothelial function or processed for microscopy as described previously. 115

In the standard procedure for specular microscopy isolated corneas were perfused for $6 \mathrm{~h}$ at $34^{\circ} \mathrm{C}$ with glutathione bicarbonate Ringers' solution (GBR) as previously described." In some cases, as indicated below, alternative perfusion solutions were used to assess particular aspects of stromal swelling and hydration during specular microscopy. The ionic composition of these various solutions is given in Table 1, and the precise formulation of CPTES in terms of both the weight and millimolar concentrations of the constituent salts and other chemicals is provided in Table 2 . 
Table 1 Composition of solutions (mmol/l)

\begin{tabular}{|c|c|c|c|c|c|}
\hline & $\begin{array}{l}\text { Rabbit aqueous } \\
\text { humour* }\end{array}$ & $G B R$ & CPTES & GBR/TES & $B C F R$ \\
\hline $\mathrm{Na}^{+}$ & $143 \cdot 0$ & $158 \cdot 48$ & 31 & $129 \cdot 82$ & $151 \cdot 4$ \\
\hline $\mathrm{K}^{+}$ & $4 \cdot 7$ & $4 \cdot 82$ & 100 & $4 \cdot 82$ & 6.75 \\
\hline $\mathrm{Cl}$ & $107 \cdot 9$ & $136 \cdot 77$ & 32 & $38 \cdot 44$ & $149 \cdot 87$ \\
\hline $\mathrm{HCO}_{3}$ & $31 \cdot 9$ & $29 \cdot 16$ & 30 & $29 \cdot 16$ & - \\
\hline $\mathrm{H}_{2} \mathrm{pO}_{4}$ & 0.7 & 0.66 & 1 & $0 \cdot 66$ & 1 \\
\hline $\mathrm{HPO}_{4}{ }^{2}$ & - & - & - & - & $4 \cdot 2$ \\
\hline $\mathrm{SO}_{4}^{2}$ & $0 \cdot 6$ & - & 0.78 & 0.78 & $0 \cdot 6$ \\
\hline $\mathrm{Mg}^{2} \cdot$ & 0.5 & 0.78 & 0.78 & 0.78 & $0 \cdot 6$ \\
\hline $\mathrm{Ca}^{2}$ & $1 \cdot 4$ & $1 \cdot 03$ & $1 \cdot 03$ & $1 \cdot 03$ & 0.56 \\
\hline Glucose & $5 \cdot 6$ & 4.99 & $5 \cdot()$ & 4.99 & $4 \cdot 45$ \\
\hline Reduced glutathione & - & 0.29 & $(0.29)^{\dagger}$ & $0 \cdot 29$ & $1 \cdot 0$ \\
\hline Adenosine & - & $0 \cdot 50$ & $(0.50) \dagger$ & $0 \cdot 50$ & - \\
\hline TES & - & - & 100 & 100 & - \\
\hline Osmolality (mosm.kg ') & 310 & 320 & 301 & 312 & 310 \\
\hline $\mathrm{pH}$ & - & $7 \cdot 4$ & $7 \cdot 7$ & $7 \cdot 6$ & $7 \cdot 4$ \\
\hline
\end{tabular}

TES: N-tris (hydroxymethly)methyl-2-aminoethane sulphonate. Application of the Henderson-Hasselbalch equation for the dissociation of this zwitterionic buffer in the CPTES medium shows that $62 \%$ of the TES is in the anionic form.

GBR: Glutathione bicarbonate Ringer's solution.

CPTES: Potassium-rich, TES buffered corneal preservation solution."

GBR/TES: GBR with $100 \mathrm{mmol} / \mathrm{Cl}$ replaced with TES.

BCFR: Bicarbonate-free Ringer's solution.

*From Kinsey." + Ingredients given in parentheses are normally absent but included in specific experiments as indicated.

The structural integrity of the endothelial layer was assessed by light microscopy after vital staining with trypan blue and alizarin red S. ${ }^{21}$ Ultrastructural examination of the endothelium in some stored corneas was carried out using transmission electron microscopy as described previously. ${ }^{15}$

\section{STORAGE CONDITIONS}

Endothelial integrity was evaluated after storage for either one or three days at $0^{\circ} \mathrm{C}$ in either GBR or CPTES solutions having the compositions given in Table 1. Additional groups of corneas were stored for

Table 2 Formulation of CPTES: a hyperkalaemic preservation medium for the cornea

\begin{tabular}{lcr}
\hline Ingredient & g/l or ml/l & mmol/l \\
\hline $\mathrm{NaHCO}_{3}$ & $0.520 \mathrm{~g}$ & 29.996 \\
$\mathrm{NaH}_{2} \mathrm{PO}_{4} .2 \mathrm{H}_{2} \mathrm{O}$ & $0.156 \mathrm{~g}$ & 1.000 \\
$\mathrm{MgSO}_{4} .7 \mathrm{H}_{2} \mathrm{O}$ & $0 \cdot 192 \mathrm{~g}$ & 0.780 \\
$\mathrm{CaCl}_{2} .6 \mathrm{H}_{2} \mathrm{O}$ & $0 \cdot 226 \mathrm{~g}$ & 1.030 \\
$\mathrm{Glucose}$ & $0.901 \mathrm{~g}$ & 5.000 \\
$\mathrm{TES}^{*}$ (free acid) & $22.920 \mathrm{~g}$ & 100.000 \\
Potassium hydroxide (1 N KOH stock) & $100 \mathrm{ml}$ & 100.000 \\
Hydrochloric acid (1 N HCl stock) & $30 \mathrm{ml}$ & 30.000 \\
\hline
\end{tabular}

Total osmolality $=301 \mathrm{mosm} / \mathrm{kg}$. $\mathrm{pH}=7 \cdot 7$ after gassing with $\mathrm{O}_{2} / \mathrm{CO}_{2}$ $(95 \% / 5 \%)$.

*TES: N-Tris (hydroxymethyl)methyl-2-aminoethane sulphonic acid (crystalline free acid; Sigma Product No. T-1375). three days in similar solutions having the following minor modifications to their formulation:

CPTES without the addition of $\mathrm{CaCl}_{2}$; CPTES with the addition of adenosine and reduced glutathione in the concentrations normally present in GBR; bicarbonate-free GBR and bicarbonate-free CPTES in which the $\mathrm{NaHCO}_{3}(30 \mathrm{mmol} / \mathrm{l})$ was replaced with $30 \mathrm{mmol} / \mathrm{l} \mathrm{NaCl}$; and finally GBR/TES in which $100 \mathrm{mmol} / \mathrm{l}$ of chloride in the standard GBR solution was replaced with $100 \mathrm{mmol} / \mathrm{l}$ TES. One additional group of corneas was stored in CPTES at $0^{\circ} \mathrm{C}$ for five days.

ANALYSIS

As in previous studies, ${ }^{115}$ measurements of stromal thickness for individual corneas were scheduled at the same time intervals during perfusion, and the change in thickness was normalised with respect to initial values determined immediately after enucleation. This procedure enabled mean percentage changes $( \pm 1$ SEM $)$ in stromal thickness for a group of corneas treated in a particular way to be evaluated as a mean normalised response curve. The rate of stromal swelling or thinning during perfusion was calculated from a regression analysis of the response curves to provide a measure of the efficiency of the endothelia in controlling stromal hydration. The Tukey-Kramer method for unplanned comparisons was used as described by Sokal and Rohlf ${ }^{22}$ for comparing regression coefficients in this analysis. 


\section{Results}

\section{EXTENT OF CORNEAL SWELLING}

DURING STORAGE

In all experiments the initial thickness of each cornea was measured in situ before dissection by mounting the enucleated eye under the specular microscope. The mean thickness of the corneas used in these experiments was $317 \mu \mathrm{m}(\mathrm{SD}= \pm 23)$.

Fig. 1 shows the mean increase in stromal thickness sustained by each of the groups of corneas during storage at $0^{\circ} \mathrm{C}$ when the metabolic pumps responsible for normal control of tissue hydration are impaired or retarded. Corneas stored overnight in GBR swell to the same extent as that reported for rabbit corneas that were stored in moist chambers without excision from the whole eye. Under these conditions the endothelium was bathed throughout with aqueous humour. ${ }^{23} 24$

Corneas stored for the same length of time in CPTES (with or without $\mathrm{Ca}^{+}$), however, swell only about half as much; that is, corneas stored in CPTES were significantly thinner than those stored in GBR $(p<0.005)$. Similarly, after three days' storage the swelling of corneas bathed in CPTES was significantly less than for the corresponding group of corneas stored in GBR $(p<0 \cdot 001)$. The omission of calcium or the addition of adenosine and reduced glutathione did not influence the degree of swelling in corneas stored in CPTES for three days at $0^{\circ} \mathrm{C}$ (Fig. 1). The omission of bicarbonate, however, led to higher mean thicknesses for groups of corneas stored in either CPTES or GBR. Corneas stored in GBR/TES swelled significantly less than corneas stored in normal GBR $(\mathrm{p}<0.05)$; the extent of swelling was equivalent to storage in CPTES. Fig. 1 also shows that corneas stored in CPTES do not double in thickness until after five days' storage, whereas corneas stored in GBR attain this degree of swelling in only three days.

\section{MORPHOLOGY}

\section{Light microscopy}

The structural integrity of corneal endothelium was retained following storage in CPTES for three and five days at $0^{\circ} \mathrm{C}$. Examples of the intact endothelia observed both immediately after five days' storage at $0^{\circ} \mathrm{C}$ and after a further four hours' perfusion at $34^{\circ} \mathrm{C}$ are shown in Fig. 2A and B respectively.
Fig. 1 Changes in stromal thickness during storage for corneas bathed in various solutions at $0^{\circ} \mathrm{C}$. The mean $( \pm 1$ SEM) increase in thickness and the number of corneas measured in each group are represented in each histogram. Data for corneas stored in aqueous humour were taken from Sherrard. ${ }^{23}{ }^{3+}$ Statistically different levels of corneal swelling are indicated (Student's $t$-test).

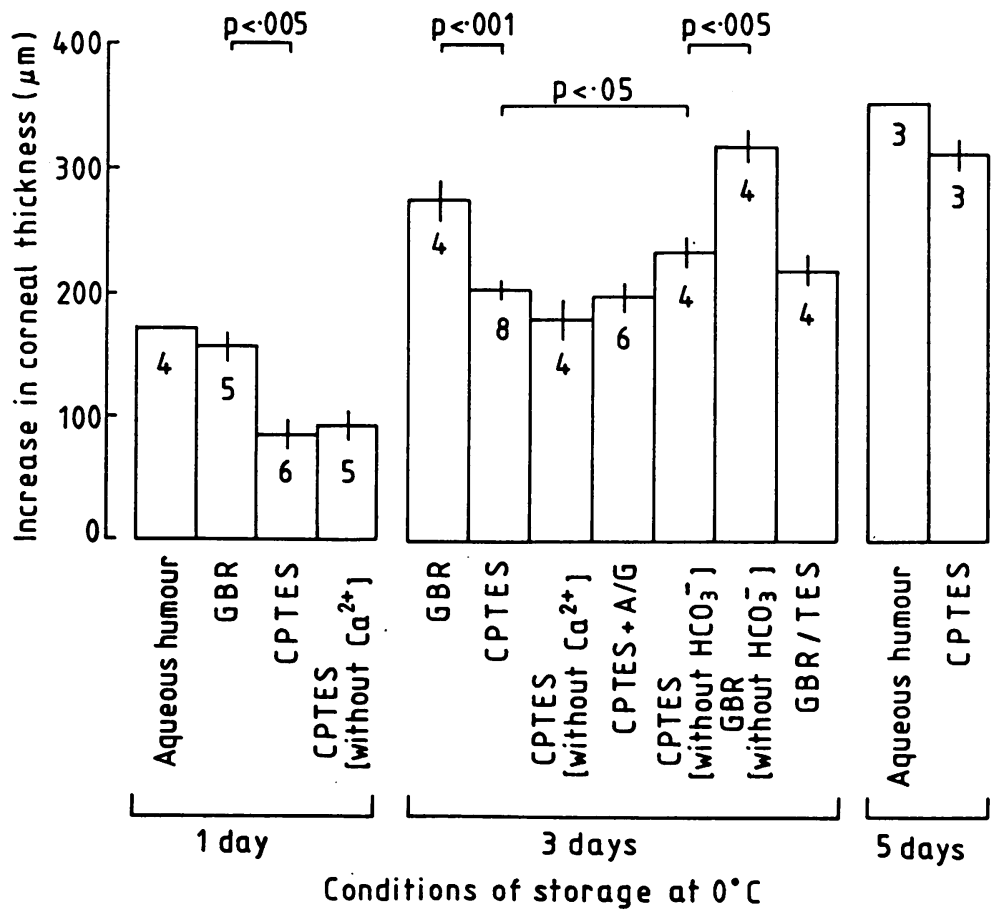



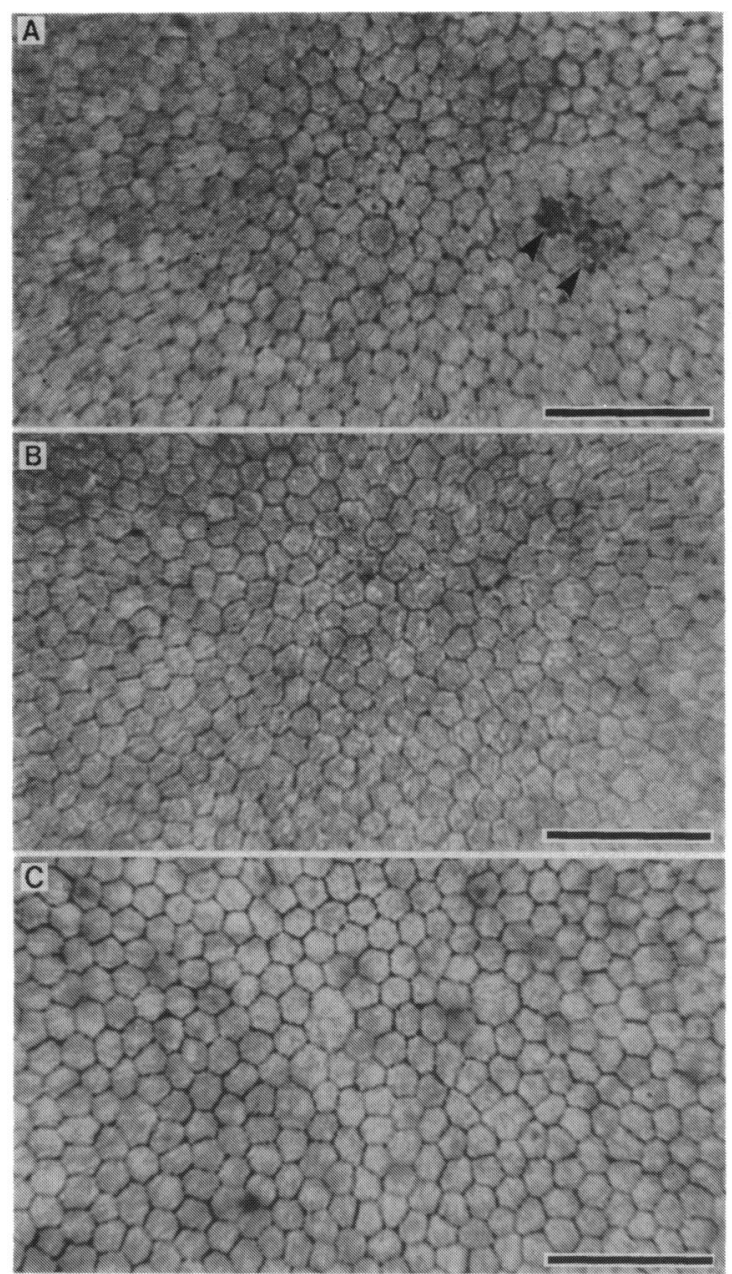

Fig. 2 Light microscopy of corneal endothelium after staining with trypan blue and alizarin red $S$. A: Specimen stained immediately after five days storage in CPTES at $0^{\circ} \mathrm{C}$. Very few areas of endothelial damage could be located on corneas stored in this way; a small isolated group of 'damaged' cells is arrowed. B: Specimen stored for five days in CPTES at $0^{\circ} \mathrm{C}$ and stained after a further four hours perfusion with $G B R$ at $34^{\circ} \mathrm{C}$ showing a completely intact endothelium, and none of the cells were permeable to trypan blue. C: Fresh cornea perfused with GBR/TES for six hours at $34^{\circ} \mathrm{C}$. Scale bar is $100 \mu \mathrm{m}$.

\section{Ultrastructure}

After three days' storage in either GBR or CPTES corneas showed a normal endothelium in close apposition to Descemet's membrane (Fig. 3A,B). There was some slight oedema localised within the interdigitated cellular processes of the basal region after both treatments, while samples stored in GBR also showed slight swelling of rough endoplasmic
A
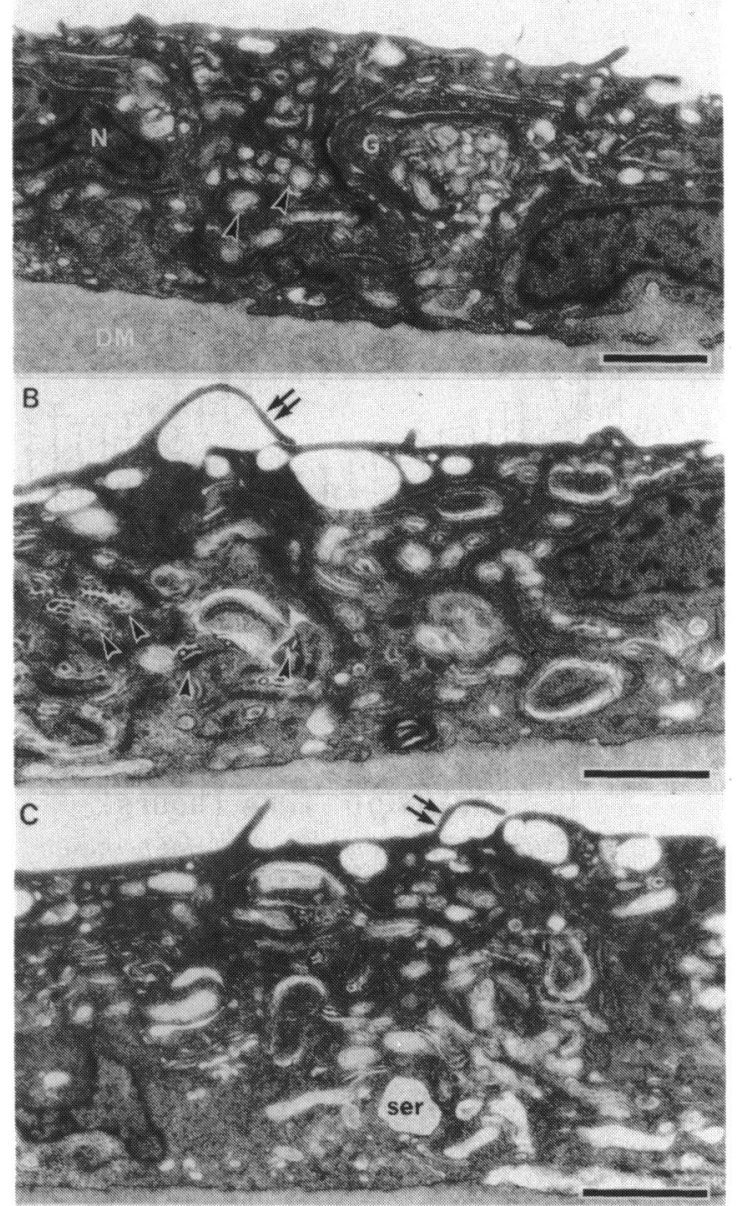

Fig. 3 Endothelial ultrastructure following storage at $0^{\circ} \mathrm{C}$. A: Three days' storage in GBR; endothelium is closely apposed to Descemet's membrane (DM) and shows invaginated nuclei $(\mathrm{N})$, a well developed Golgi apparatus (G), and slight swelling of some mitochondria (arrowed). B and C: Three and five days respectively in CPTES; mitochondria show moderate swelling with diffuse pallid matrices as well as condensed and beaded forms (arrow heads), vesiculated smooth endoplasmic reticulum (ser), and raised apical flaps (double arrows). Apical junctions are closed. Scale bar is $1 \mu \mathrm{m}$.

reticulum, increased invagination of the nucleus, and a distinct profileration of Golgi vesicles. Mitochondria showed similar reversible changes in corneas stored in both GBR and CPTES.

After five days' storage in CPTES (Fig. 3C) the endothelium was moderately swollen, and in a small number of samples there was minor localised detachment from Descemet's membrane, though apical 


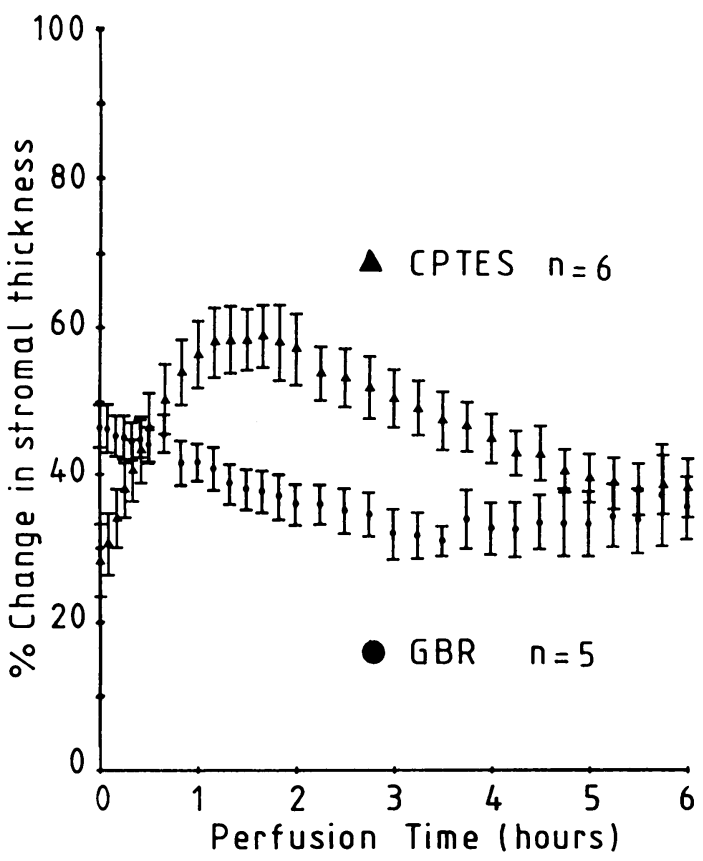

Fig. 4 Mean stromal hydration changes during perfusion for groups of corneas after storage for one day in either CPTES $(\mathbf{\Delta})$ or $G B R(\bullet)$ at $0^{\circ} \mathrm{C}$. The biphasic curve for corneas stored in CPTES is explained in the text; these corneas thinned at $5 \cdot 6($ SEM $0 \cdot 2) \%$ per hour. Corneas stored in GBR thinned at $4 \cdot 8$ (SEM 0.2)\% per hour. Sherrard has previously shown that the criterion for a viable cornea is a 'temperature reversal' rate of $25 \mu \mathrm{m}$ in two hours $(\sim 4 \%$ per hour). ${ }^{24}$

junctions remained closed. Most organelles showed similar changes to those seen after three days' storage, though now smooth endoplasmic reticulum was swollen and vesiculated. The state of the endothelium after storage for three days in GBR and up to five days in CPTES would suggest that, immediately after storage, no irreversible changes had occurred to the majority of cells.

\section{CHANGES IN STROMAL HYDRATION DURING PERFUSION}

Fig. 4 shows contrasting responses for corneas stored in CPTES compared with those stored in GBR. The marked difference in stromal thickness at the beginning of perfusion following storage at $0^{\circ} \mathrm{C}$ is clearly apparent; thereafter corneas which had been bathed in GBR during storage decrease in thickness during subsequent perfusion at $34^{\circ} \mathrm{C}$. This response is commonly referred to as the 'temperature reversal' response, since it describes the characteristic reversal of swelling by the reactivation of endothelial-pump activity when the tissue is rewarmed to physiological temperature. Corneas stored in CPTES did not 'temperature reverse' immediately, but showed a biphasic response in which they initially underwent further swelling during the first 90 minutes of perfusion. Thereafter, they decreased in thickness by $5 \cdot 6$ (SEM $0.2 \%$ ) per hour, demonstrating the activity of fully functional endothelia. Similarly, Fig. 5A shows that, for the group of corneas stored for three days in CPTES at $0^{\circ} \mathrm{C}$ an identical rate of thinning was preceded by stromal swelling for 90 minutes when these corneas were perfused with GBR at $34^{\circ} \mathrm{C}$. An additional group of corneas were stored in CPTES for three days and then perfused at $34^{\circ} \mathrm{C}$ for two hours with GBR followed by bicarbonate-free medium (BCFR) for a further four hours. Fig. 5B shows that during the second phase of perfusion, when the bicarbonate pump was switched off, these corneas did not thin but swelled further, thus confirming that the deturgescence recorded during the second phase for those corneas perfused throughout with GBR (Fig. 5A) was due entirely to active endothelial pumping.

When corneas stored under identical conditions were perfused with GBR/TES instead of GBR they did not swell at the beginning of the perfusion. Fig. 5A shows that these corneas began thinning immediately and eventually reached a plateau at a constant thickness throughout the remaining period of perfusion. The role of a functioning endothelium in controlling the hydration of these corneas is further demonstrated in Fig. 6 by induced swelling when the preparation was perfused with medium that does not contain $\mathrm{CO}_{2}$ or bicarbonate ions (BCFR/ TES). ${ }^{1 *}$ Under these conditions the bicarbonatedependent pump is inactivated and the cornea swells at $25 \mu \mathrm{m} / \mathrm{h}$. This swelling was reversed at the same rate when the cornea was again perfused with GBR/ TES. Fresh corneas perfused with GBR/TES alone for six hours retained the integrity of the endothelial layer as illustrated in Fig. 2C.

Corneas stored for three days in GBR at $0^{\circ} \mathrm{C}$ showed a normal 'temperature reversal' response when perfused with GBR at $34^{\circ} \mathrm{C}$ (Fig. 7). However, corneas stored at $0^{\circ} \mathrm{C}$ for three days in GBR containing $100 \mathrm{mmol} / \mathrm{l}$ TES (GBR/TES) showed a biphasic response during subsequent perfusion with GBR at $34^{\circ} \mathrm{C}$ that was similar to that of corneas stored in CPTES and perfused with GBR. Fig. 7 shows that initially the stroma swelled for $90 \mathrm{~min}$ before an active dehydrating mechanism was apparent.

Corneas stored for three days at $0^{\circ} \mathrm{C}$ in CPTES containing the same concentrations of glutathione and adenosine as in GBR, or stored in CPTES with the omission of calcium, showed identical responses during perfusion as the group of corneas stored in the 

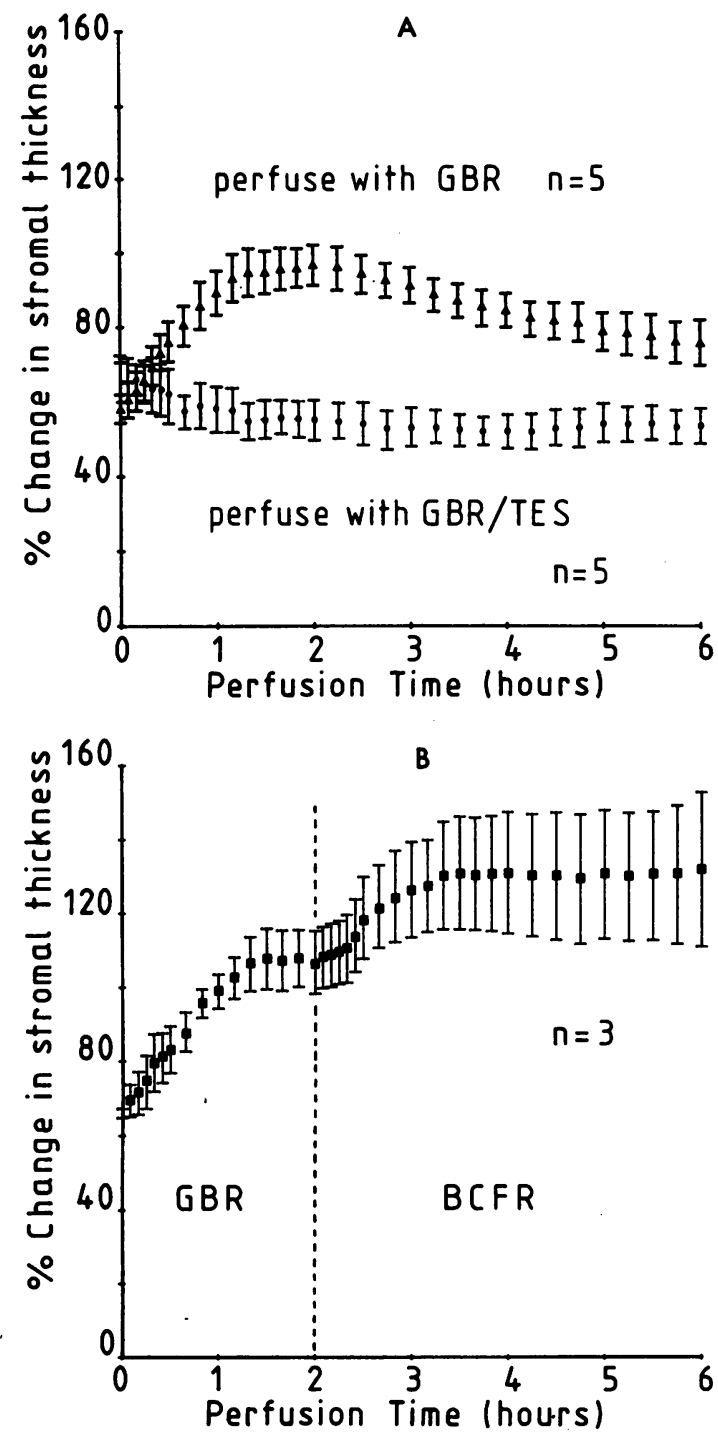

Fig. 5 Stromal thickness changes for corneas stored in CPTES for three days at $0^{\circ} \mathrm{C}$. A: Mean response curves for groups of corneas perfused at $34^{\circ} \mathrm{C}$ under the specular microscope with either $G B R(\Delta)$ or $G B R / T E S(\bullet)$.

B: Mean response curve for a group of corneas when perfusion with $G B R$ was changed after two hours to perfusion with bicarbonate-free Ringer's solution (BCFR) as indicated by the broken line.

standard CPTES solution. Fig. 8 shows that the stromal thickness curves can be superimposed, having the same biphasic profile as that shown in Fig. 5A for corneas stored in CPTES. Corneas stored in CPTES containing $\mathrm{Ca}^{++}$thinned at 5.6 (SEM 0.2)\% per hour $(n=5)$, which was not significantly different from the

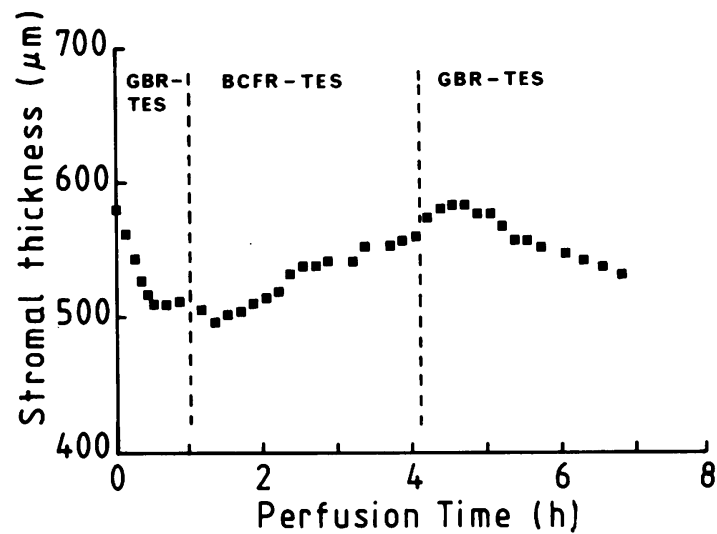

Fig. 6 Endothelial pump activity in a cornea stored in $C P T E S$ for three days at $0^{\circ} \mathrm{C}$. Perfusion with GBR/TES was interrupted by perfusion with BCFR/TES which contained no bicarbonate or $\mathrm{CO}_{2}$. Broken lines represent the points at which solutions were changed. See text for interpretation of these curves.

group of corneas stored in CPTES without calcium (5.9 (SEM 0.2)\% per hour, $n=4)$. However, the corneas stored in CPTES with adenosine and glutathione thinned at $6 \cdot 4($ SEM $0 \cdot 1) \%$ per hour $(n=6)$ which was not significantly different from the $\mathrm{Ca}^{2+}$ -

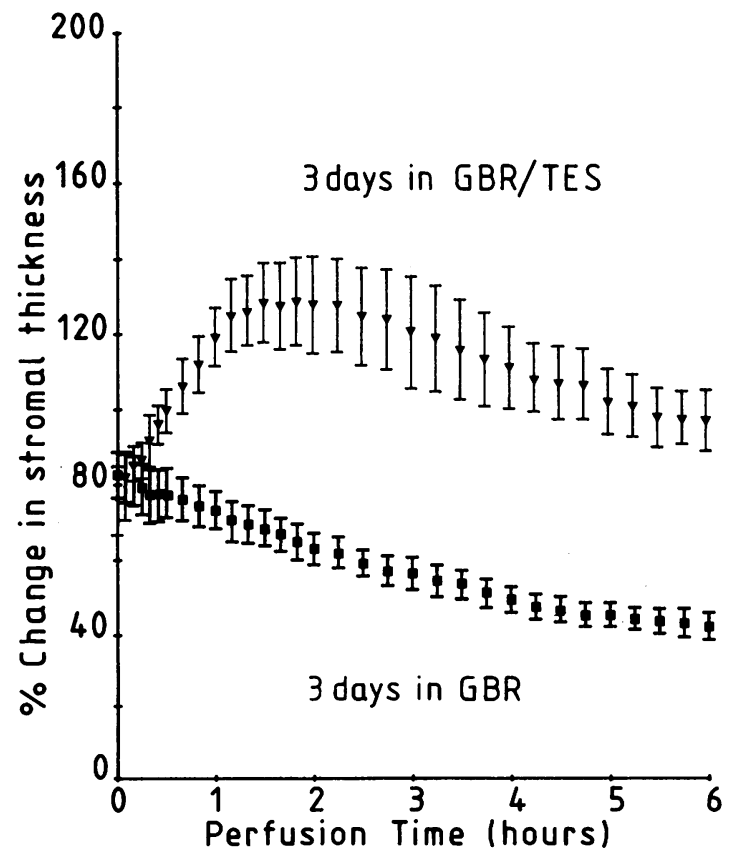

Fig. 7 Mean stromal thickness curves for groups of corneas stored for three days at $0^{\circ} \mathrm{C}$ in either $G B R(\square), n=4$; or $G B R / T E S(\nabla), n=3$. Both groups of corneas were perfused with $\mathrm{GBR}$ at $34^{\circ} \mathrm{C}$. 


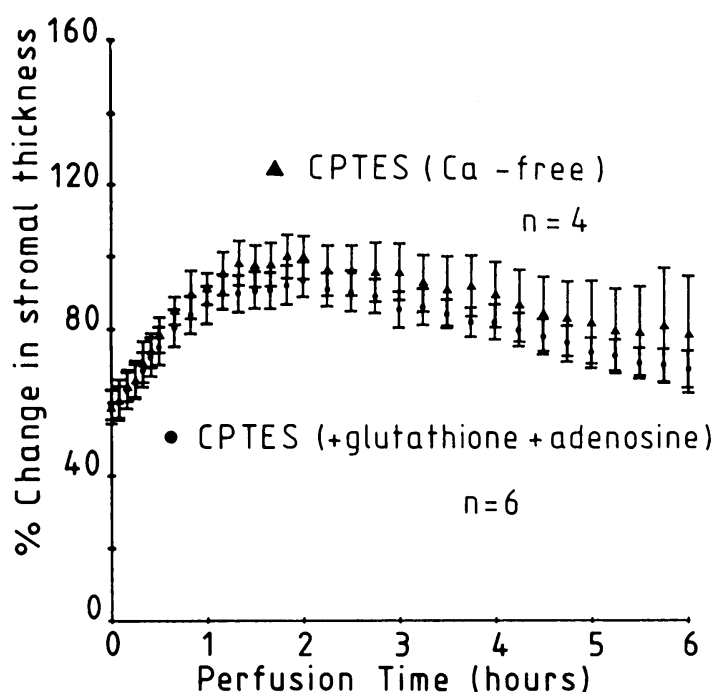

Fig. 8 Comparison of the mean stromal thickness curves derived for groups of corneas after storage for three days at $0^{\circ} \mathrm{C}$ in either calcium-free CPTES $(\mathbf{\Delta})$ or CPTES containing glutathione and adenosine $(\bullet)$. All corneas were perfused at $34^{\circ} \mathrm{C}$ with $G B R$.

free group but was significantly different from the group stored in CPTES when compared at the $95 \%$ level but not the $99 \%$ level of confidence (Table 3 ).

The endothelium of corneas stored for three days in solutions from which bicarbonate was omitted dehydrated the swollen stroma at a greater rate than the comparable groups of corneas stored in equiv- alent solutions containing bicarbonate. Table 3 shows that the maximum rate of stromal dehydration was observed in the group of corneas stored in bicarbonate-free CPTES. The Tukey-Kramer analysis, comparing the regression coefficients of all groups, shows that the rate of stromal thinning was significantly greater after storage in bicarbonate-free CPTES than after storage for three days in any of the other solutions $(\mathrm{p}<0 \cdot 01)$.

Corneas stored for five days in CPTES also showed a distinctive biphasic curve for the proportional change in stromal thickness during perfusion. Fig. 9 shows that this group of corneas swelled by $100 \%$ of their initial thickness during the storage interval and by a further $47 \%$ during the initial two hours of perfusion; therafter these corneas thinned very efficiently at $15 \cdot 2($ SEM 0.5$) \%$ per hour.

\section{Discussion}

\section{IONIC COMPOSITION OF THE PRESERVATION}

\section{SOLUTION}

Hypothermic preservation of corneas at refrigerator temperature $\left(4^{\circ} \mathrm{C}\right)$ provides the basis of tissue storage for clinical use today. Most storage solutions in popular use are based on a conventional tissue culture medium to provide ionic support for the tissue during the preservation period. These media are specifically designed to maintain the metabolic status of cells during in-vitro incubation at normothermic temperatures. However, it has been suggested that, with the application of hypothermia,

Table 3 Regression coefficients (slopes) of stromal thickness curves and comparisons of the differences between slopest

\begin{tabular}{|c|c|c|c|c|c|c|c|}
\hline \multirow[b]{2}{*}{ Storage solution } & \multicolumn{7}{|c|}{ Difference between slopest } \\
\hline & $A$ & $B$ & $C$ & I) & $E$ & $F$ & $\begin{array}{l}\text { Regression } \\
\text { coefficient } \\
( \pm S E)\end{array}$ \\
\hline A. GBR & - & 0.66 & $0 \cdot 31$ & $1 \cdot 11^{*}$ & $4 \cdot 19^{* * *}$ & $2 \cdot 97^{* *}$ & $\begin{array}{l}-5 \cdot 26 \pm 0 \cdot 26 \\
n=4\end{array}$ \\
\hline B. CPTES (without Ca") & $\begin{array}{c}1 \cdot 10 \\
(1 \cdot 39)\end{array}$ & - & 0.34 & 0.46 & $3 \cdot 53^{* * *}$ & $2 \cdot 31^{* * *}$ & $\begin{array}{l}-5 \cdot 91 \pm(0 \cdot 23 \\
n=4\end{array}$ \\
\hline C. CPTES & $\begin{array}{l}1 \cdot() 2 \\
(1 \cdot 28)\end{array}$ & $\begin{array}{c}0.96 \\
(1 \cdot 20)\end{array}$ & - & $(1) .80 *$ & $3 \cdot 88^{* * * *}$ & $2 \cdot 65^{* * *}$ & $\begin{array}{l}-5 \cdot 57 \pm(0 \cdot 19 \\
\mathrm{n}=5\end{array}$ \\
\hline $\begin{array}{c}\text { D. CPTES (+glutathione) } \\
\text { (+adenosine) }\end{array}$ & $\begin{array}{l}0.93 \\
(1 \cdot 16)\end{array}$ & $\begin{array}{l}0 \cdot 86 \\
(1 \cdot 07)\end{array}$ & $\begin{array}{c}(0.74 \\
(0.93)\end{array}$ & - & $3 \cdot\left(08^{* * *}\right.$ & $1 \cdot 85 * *$ & $\begin{array}{l}-6 \cdot 37 \pm(0 \cdot 1.3 \\
n=6\end{array}$ \\
\hline E. CPTES (without $\mathrm{HCO}_{3}$ ) & $\begin{array}{l}0.93 \\
(1 \cdot 16)\end{array}$ & $\begin{array}{c}0 \cdot 86 \\
(1 \cdot 07)\end{array}$ & $\begin{array}{l}(0.74 \\
(0.93)\end{array}$ & $\begin{array}{l}0.61 \\
(0.76)\end{array}$ & - & $1 \cdot 22 *$ & $\begin{array}{l}-9 \cdot 45 \pm(0 \cdot 13 \\
n=5\end{array}$ \\
\hline F. GBR (without $\mathrm{HCO}_{3}$ ) & $\begin{array}{l}1 \cdot 21 \\
(1.51)\end{array}$ & $\begin{array}{l}1 \cdot 15 \\
(1 \cdot 44)\end{array}$ & $\begin{array}{l}1 \cdot(1) \\
(1 \cdot 34)\end{array}$ & $\begin{array}{l}0.98 \\
(1.23)\end{array}$ & $\begin{array}{l}(0.98 \\
(1.23)\end{array}$ & - & $\begin{array}{l}-8 \cdot 22 \pm(0 \cdot 2 x \\
n=5\end{array}$ \\
\hline
\end{tabular}

†The table is presented as a matrix which allows comparison of the regression data between any two of the experimental groups. The calculated differences between slopes are tabulated in the upper section and these are conveniently compared with the minimum significant difference calculated by the Tukey-Kramer statistical method"' and given in the lower section ( $p=() \cdot(0)$ and values in parentheses $\mathrm{p}=(0.01)$. Where appropriate differences between slopes that are statistically significant are indicated $(* \mathrm{p}<0) \cdot(0)$ and $* *{ }^{*} \mathrm{p}<() \cdot(01)$.

$\ddagger$ Rate of change of stromal thickness (\% per $h$ ). Negative values indicate corneal thinning. 


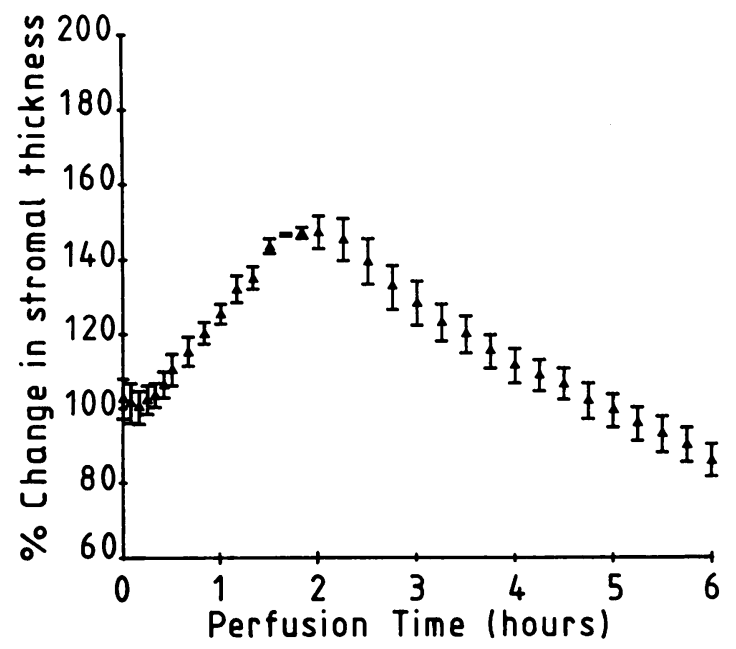

Fig. 9 Stromal hydration changes during normothermic perfusion for a group of corneas after storage in CPTES for five days at $0^{\circ} \mathrm{C}(n=3)$.

it is unnecessary to support metabolism: control of ion and water distribution between the intracellular and extracellular compartments can be achieved by physical, not biochemical, means. Since the driving force for sodium uptake and potassium depletion is the different ionic balance in extracellular fluid, and since the driving force for net fluid entry is the impermeant intracellular constituents, both changes can be prevented by appropriate alteration to the composition of the extracellular fluid. This is the basis for the design of so-called 'wash-out' solutions used for hypothermic preservation of organs such as kidneys ${ }^{1}$ and is also the rationale that we have presented previously for the formulation of CPTES, a hyperkalaemic solution for the preservation of corneas."

\section{REDUCED CORNEAL SWELLING DURING} STORAGE IN CPTES

This study showed that CPTES was more effective than glutathione bicarbonate Ringer's (GBR) solution in restricting stromal swelling during hypothermic storage. Predictably, corneas stored in GBR swelled to the same extent as that reported for tissue stored in the intact eye where the endothelium was bathed throughout with aqueous humour. ${ }^{23+4}$ The effectiveness of CPTES in controlling stromal swelling during storage was shown to be due to the presence of the zwitterionic buffer, TES, since incorporation of an equivalent concentration of TES into the GBR (that is, GBR/TES) produced a similar effect.
BIPHASIC HYDRATION CHANGES DURING PERFUSION

Corneas stored in CPTES showed a characteristic biphasic stromal thickness curve during perfusion which contrasted with the more usual 'temperature reversal' curve shown by the corneas stored in GBR. This again reflects an osmotic response due to the presence of the ampholytic molecule, TES, which permeates into the stroma during storage and establishes an osmotic gradient when the tissue is perfused with GBR. This osmotic gradient, leading to further corneal swelling, was observed to persist consistently for $80-90 \mathrm{~min}$, and presumably reflects the time necessary for complete elution of the TES from the stroma and re-establishment of osmotic equilibrium. Thereafter the presence of an active dehydrating endothelial pump was manifest by a decrease in stromal thickness during the remaining perfusion period. In contrast, the group of corneas perfused with bicarbonate-free medium during this second phase swelled further, thus confirming that endothelial pumping was totally responsible for thinning in those corneas perfused throughout with GBR. Furthermore, the demonstration of reversible swelling during interrupted perfusion with BCFR verified the presence of a bicarbonate-dependent endothelial pump which was responsible for controlling stromal dehydration. The role of TES in generating the initial swelling period was confirmed by repeating the storage experiments with corneas that were subsequently perfused with GBR/TES which contained the same concentration of TES $(100 \mathrm{mM})$ as the storage medium. In these cases the corneas did not swell at the beginning of perfusion but began thinning immediately. Furthermore, corneas stored in GBR/TES and perfused with normal GBR also showed a biphasic curve compatible with that shown by corneas stored in CPTES.

METABOLIC INFLUENCE OF SOME OTHER IONS While the omission of calcium from CPTES, which has been discussed in a previous publication, " or the addition of adenosine and glutathione to the CPTES preservation medium did not greatly influence the viability of the endothelium during hypothermic storage, the omission of bicarbonate resulted in more efficient deturgescence of the stored tissue. It has already been explained that during hypothermic storage it is unlikely that normal metabolic support will be required for optimum preservation of corneal endothelium. However, the role of bicarbonate may have special significance for the preservation of this tissue. In studies establishing the role of bicarbonate ions for the function of the electrogenic dehydrating pump of corneal endothelium, Hodson demonstrated a significant benefit from omitting $\mathrm{HCO}_{3}$ from 
corneal bathing solutions. ${ }^{25} \mathrm{He}$ postulated that storage in bicarbonate-free medium prevented the pump from being expended during storage and would therefore prolong the in-vitro life of the tissue. Furthermore, Mayes et al also demonstrated the deleterious effect of exogenous bicarbonate on the subsequent functions of rabbit corneal endothelium after prolonged refrigerated storage..$^{20}$

One consequence of storing tissue in bicarbonatefree medium would be the lack of effective protonbuffering. However, the incorporation of $100 \mathrm{mM}$ TES in CPTES provides efficient $\mathrm{pH}$ buffering in the absence of bicarbonate should this prove to be important for improved hypothermic storage of corneas. ${ }^{12}{ }^{13}$ TES is one of a family of sulphonic acid derivatives having high solubility and low chemical toxicity which have been used very effectively as biological buffers. ${ }^{12-14272 \times}$ As a pH buffer (for example, in tissue culture applications) these zwitterionic compounds are generally used at a concentration in the range $10-25 \mathrm{mM}$. However, as described previously, ${ }^{11} 12100 \mathrm{mmol} / \mathrm{l} \mathrm{TES}$ is included in the preservation solution to act as an impermeant anion during hypothermic preservation, and this concentration of sulphonic acid buffer is known to be tolerated by a variety of cells, tissues and organs. ${ }^{1122724}$ Garlick et al ${ }^{24}$ discovered a positively beneficial effect of supplementing Krebs-Henseleit buffer solution with $100 \mathrm{mM}$ HEPES (N-2-hydroxyethyl) piperazine- $\mathrm{N}^{\prime}$-2-ethane sulphonic acid) for the control of acidosis in perfused hearts. They concluded that both the rate and extent of cellular acidification are significantly reduced when $100 \mathrm{mM}$ HEPES was included and also that this concentration of HEPES has a significant protective effect on the ischaemic myocardium. ${ }^{29}$ In the present study the experiments in which preserved corneas were perfused with GBR/ TES showed that corneal endothelium remains metabolically active and fully functional during six hours' perfusion in the presence of $100 \mathrm{mmol} / \mathrm{l} \mathrm{TES}$ at physiological temperatures. The structural integrity of the endothelium was also confirmed after six hours' perfusion with GBR/TES (Fig. 2C). It is concluded, therefore, that $100 \mathrm{mM}$ TES has no detectable detrimental effects on the structural and functional integrity of corneal endothelium either at normal or at reduced temperature. On the contrary, storage of corneas in the 'intracellular-type' solution, CPTES, was clearly beneficial for restricting stromal swelling during hypothermic storage and yielding corneas that could deturgesce more efficiently than after storage in the 'extracellular-type' solution, GBR.

Further benefits of preventing corneal swelling during hypothermic storage in CPTES are realised by incorporating colloid osmotic agents such as polyvinylpyrrolidone or chondroitin sulphate, as described elsewhere. ${ }^{11} 14$

We are grateful to Mr David Chapman, who provided excellent technical help for these studies.

\section{References}

1 Pegg DE. Principles of tissue preservation. In: Morris PJ, Tilney NL, eds. Progress in transplantation. Edinburgh: Churchill Livingstone, 1985: 69-105.

2 Taylor MJ. Clinical cryobiology of tissues: preservation of corneas. Cryobiology 1986: 23: 323-53.

3 Bito LZ, Salvador EV. Intraocular fluid dynamics II: Postmortem changes in solute concentrations. Exp Eye Res 1970; 10: 273-87.

4 De Roeth A Jr. Metabolism of the stored cornea. Arch Ophthalmol 1950; 44: 659-65.

5 Hull DS, Green K, Bowman K. Csukas S, Riley MV. Intracellular $\mathrm{pH}$ and glutathione levels in rabbit corneal endothelium following storage in moist chamber and MK medium. Invest Ophthalmol Vis Sci 1983; 24: 214-7.

6 McCarey BE, Kaufman HE. Improved corneal storage. Invest Ophthalmol Vis Sci 1974; 13: 165-73.

7 Schimmelpfennig B. Corneal preservation at $-4^{\circ} \mathrm{C}$ in a modified tissue culture medium. Graefes Arch Clin Exp Ophthalmol 1978: 206: $57-66$.

8 Kaufman HE, Varnell ED, Kaufman S. Chondroitin sulfate in a new cornea preservation medium. Am J Ophthalmol 1984: 98: $112-4$.

9 Yau CW, Kaufman HE. A medium term corneal preserving medium (K-SOL). Arch Ophthalmol 1986; 104: 598-60)1.

10 Bourne WM. Endothelial cell survival on transplanted human corneas preserved at $4^{\circ} \mathrm{C}$ in $2.5 \%$ chondroitin sulfate for 1 to 13 days. Am J Ophthalmol 1986; 102: 382-6.

11 Taylor MJ, Hunt CJ. A new preservation solution for storage of corneas at low temperatures. Curr Eye Res 1985; 4: 963-73.

12 Taylor MJ. The role of $\mathrm{pH}$ buffer capacity in the recovery of function of smooth muscle cooled to $-13^{\circ} \mathrm{C}$ in unfrozen media. Cryobiology; 1982; 19: 585-601.

13 Taylor MJ, Pignat Y. Practical acid dissociation constants, temperature coefficients, and buffer capacities for some biological buffers in solutions containing dimethyl sulfoxide between 25 and $-12^{\circ} \mathrm{C}$. Cryobiology 1982; 19: 99-109.

14 Good NE, Winget GD, Winter W, Connolly TN, Izawa S, Singh RMM. Hydrogen ion buffers for biological research. Biochemistry 1966; 5: 467-77.

15 Taylor MJ, Hunt CJ. Tolerance of corneas to multimolar dimethyl sulfoxide at $0^{\circ} \mathrm{C}$ : implications for cryopreservation. Invest Ophthalmol Vis Sci 1989; 30: 400-12.

16 Dikstein S, Maurice DM. The metabolic basis to the fluid pump in the cornea. J Physiol (Lond) 1972; 221: 29-41.

17 Edelhauser HF, Van Horn DL, Hyndiuk RA, Schultz RO. Intraocular irrigating solutions: their effect on the corneal endothelium. Arch Ophthalmol 1975; 93: 648-57.

18 Taylor MJ, Hunt CJ, Madden PW. Hypothermic preservation of corneas in a hyperkalemic solution (CPTES): II. Extended storage in the presence of chondroitin sulphate. Br J Ophthalmol 1989; 73: 792-8(2)

19 Taylor MJ, Hunt CJ. The effect of polyvinyl pyrrolidone as a colloid osmotic agent in a hypothermic preservation solution for corneas. Cryo-Letters 1989; 10: 235-48.

20 Kinsey VE. The chemical composition and the osmotic pressure of the aqueous humor and plasma of the rabbit. $J$ Gen Physiol 1951: 34: 389-402.

21 Taylor MJ, Hunt CJ. Dual staining of corneal endothelium with trypan blue and alizarin red $\mathrm{S}$ : importance of $\mathrm{pH}$ for the dye-lake reaction. Br J Ophthalmol 1981; 65: 815-9.

22 Sokal RR, Rohlf FJ. Biometry: the principles and practice of statistics in biological research. 2nd ed. San Francisco: Freeman, 1981: 454-560. 
23 Sherrard ES. Method of evaluating donor cornea for transplantation. Br J Ophthalmol 1973; 57: 244-52.

24 Sherrard ES. An application of the temperature reversal effect: a possible method to evaluate donor cornea for penetrating keratoplasty. Exp Eye Res 1973; 15: 667-75.

25 Hodson S. The endothelial pump of the cornea. (Editorial.) Invest Ophthalmol Vis Sci 1977; 16: 589-91.

26 Mayes KR, Graham MV, Hodson S. The deleterious effects of exogenous bicarbonate on the rabbit cornea undergoing prolonged refrigerated storage. Exp Eye Res 1978; 26: 555-60.
27 Shipman CJ. Evaluation of 4-(2-hyroxyethyl)-1-piperazine ethane sulfonic acid (HEPES) as a tissue culture buffer. Exp Biol Med 1969; 130: 305-10.

28 Williamson JD, Cox P. Use of a new buffer in the culture of animal cells. J Gen Virol 1968; 2: 309-12.

29 Garlick PB, Radda GK. Seeley PJ. Studies of acidosis in the ischaemic heart by phosphorus nuclear magnetic resonance. Biochem J 1979; 184: 547-54.

Accepted for publication 17 April 1989. 